\title{
Research Article \\ Real-Time Gate Reassignment Based on Flight Delay Feature in Hub Airport
}

\author{
Huawei Wang, Yuxiao Luo, and Zhijian Shi \\ College of Civil Aviation, Nanjing University of Aeronautics and Astronautics, Nanjing 210016, China \\ Correspondence should be addressed to Huawei Wang; huawei678@163.com
}

Received 30 September 2013; Revised 30 October 2013; Accepted 31 October 2013

Academic Editor: Bin Yu

Copyright ( 2013 Huawei Wang et al. This is an open access article distributed under the Creative Commons Attribution License, which permits unrestricted use, distribution, and reproduction in any medium, provided the original work is properly cited.

\begin{abstract}
Appropriate gate reassignment is crucially important in efficiency improvement on airport sources and service quality of travelers. The paper divides delay flight into certain delay time flight and uncertain delay time flight based on flight delay feature. The main objective functions of model are to minimize the disturbance led by gate reassignment in the case of certain delay time flight and uncertain delay time flight, respectively. Another objective function of model is to build penalty function when the gate reassignment of certain delay time flight influences uncertain delay time flight. Ant colony algorithm (ACO) is presented to simulate and verify the effectiveness of the model. The comparison between simulation result and artificial assignment shows that the result coming from ACO is obvious prior to the result coming from artificial assignment. The maximum disturbance of gate assignment is decreased by $13.64 \%$, and the operation time of ACO is $118 \mathrm{~s}$. The results show that the strategy of gate reassignment is feasible and effective.
\end{abstract}

\section{Introduction}

Gate reassignment is a necessary procedure when its planned gate assignment is influenced by real-time operation situation and cannot be normally implemented. With the significant improvement of air transportation, gates become the key resources in airport, which are to be the bottleneck in maximizing operational efficiency. An appropriate gate assignment will make a difference in improving airport capacity and passenger satisfaction. However, in practice, disturbed by factors such as weather condition, flow control, flight schedule, and passengers, the advanced gate assignment plan cannot achieve original goal, so gate manager (or airport manager) must conduct gate reassignment timely to improve the operation effectiveness on airport surface. Rapid and effective gate reassignment plays an important role in improving operation efficiency and airport volume, decreasing operation cost of airlines, and improving service quality of passengers.

The characteristics of gate assignment based on flight delays should be described in following aspects.

(i) The difficulty in predicting the accurate flight delay, which is caused by the complicate delight delay, increases the complexity of gate assignment. Therefore, it is necessary to gate assignment on time.

(ii) Almost all the flights should be assigned proper gate under the condition of large-scale flight delay newly. The workload is very heavy, so that the traditional method of gate assignment is difficult to work effectively in gate assignment.

(iii) Large-scale flight delay can reduce the operation of airport. Constraint condition and multiobjective should be taken into account in the gate reassignment under the condition of large-scale delay in hub airport.

Considering the above characteristics of gate assignment, the intricate gate assignment under the condition of large-scale delay is a multiobject assign problem based on uncertain information.

Thus, gate reassignment is widely discussed in the worldwide research. In the way of gate preassigned, Yan and Chang [1] developed a multicommodity network flow to minimize passenger walking distances and utilized an algorithm based on the Lagrangian relaxation with subgradient methods. 
Maharjan and Matis [2] presented a binary integer multicommodity gate flow network model with the objective of minimizing the fuel burn cost of aircraft taxi by type and expected passenger discomfort. Focusing mainly on maximum gate employment, Genç et al. [3] presented a stochastic neighborhood search approach for airport assignment, which combines the benefits of heuristic approaches with some stochastic approach to building a model to minimize the number of flights assigned to aprons and the total walking distances, Cheng et al. [4] presented a metaheuristics model for airport gate assignment, which generates good solutions in a reasonable timeframe. Considering the preference and robustness in flight assignment optimization, Dorndorf et al. [5] built a model to minimize the number of trailers in gage assignment and adapted search algorithm to verify the effectiveness of the model. Some researchers focus on realtime gate assignment. Tang and Wang [6] studied airport gate assignment for airline-specific gates, which is different from the traditional gate assignment and allocated flights to airlinespecific gates is based on the perspective of the airline rather than that of the airport authority. Jaehn [7] used dynamic programming approach to building a gate assignment model based on flight number.

There are increasing researches on gate reassignment. For example, Maharjan and Matis [8] built a binary integer model for the optimal reassignment of planes to gates in response to flight delays. Gu and Chung [9] presented a genetic algorithm to minimize passenger walking distance. Some research concentrates on gate reassignment under stochastic or uncertain condition. Hou [10] built a gate reassignment model and classified flight types to minimize space and time disturbances based on flight delay feature. Tang et al. [11] built a gate reassignment model to decrease delay time and gate change, and then they calculated the disturbance by conversing delay time and designed a rapid heuristic algorithm to verify the model. Tang [12] added the influence of random factors and broadened some constraints in order to deal with delay. Yan and Tang [13] tried to build comprehensive gate assignment model at the same time based on Tang et al. [11] and Tang [12]. Some literature considered the interrelationship between planned assignment and reassignment. Şeker and Noyan [14] considered the gate assignment problem under uncertainty in flight arrival and departure times and developed stochastic programming models incorporating robustness measures based on the number of conflicting flights, idle and buffer times. Yan et al. [15] presented a simulation framework which could analyze the effects of stochastic flight delays, flexible buffer times, and real-time gate assignment rules. Some researches focused on reassignment in serious incidents such as airport closure. Taking a panoramic view of the domestic and foreign research status, most researches assigned the disturbed flight in passenger perspective. In practice, due to uncertainty and limitation of information obtained in real time, flights are possibly to be assigned more than once which will lead to increasing flight delay time and decreasing efficiency of airport resource. Therefore, both information certainty and reassignment urgency should be taken into account during gate reassignment in order to improve the efficiency of airport gates.
In the paper, the characteristics of gate reassignment based on flight delay in hub airport have been analyzed. The deposition of gate assignment and conformation of objective function are applied to reduce the complexity of research problems.

The paper divides delay flights into certain delay time flight and uncertain delay time flight base on flight delay feature. The main objective functions of model are to minimize the disturbance led by gate reassignment in the case of certain delay time flight and uncertain delay time flight, respectively. Ant colony optimization method, which is the representative method in discrete optimization, has been used to model real-time gate assignment and multiobjective optimization based on flight delay in hub airport. The remainder of paper is organized as follows. In Section 2, we describe the gate reassignment. In Section 3, we build optimization model. We design ACO to simulate the model in Section 4. In Section 5, a numerical test was performed with comparison to the artificial method. Finally, we conclude in Section 6.

\section{Gate Reassignment Description}

The purpose of gate reassignment is to obtain a new flightto-gate scheme according to scheduled assignment to reassign the delay flights in order to avoid flight conflicts in view of operation safety. Therefore, in the course of gate reassignment, certainty information, stochastic issues, and reassignment disturbance should be taken into account.

2.1. Delay Features and Information. In practice, gate managers will constantly receive estimated time information of each delay flight. From the time point of gate reassignment operation, if the estimated time of the delay flight is more close to the actual operation time, the managers will get more certain information of delay flights. That is to say the managers can schedule the delay flights actually. Otherwise, the arrival-departure information of delay flights needed by managers is not easy to be gotten correctly, so the delay feature is difficult to be holed. In the paper, the authors classified the follow-up arrival-departure flights into certain delay time flight and uncertain delay time flight. Some scheduled time node after gate reassignment is a demarcation point to reassign the follow-up arrival-departure flights into the appropriate gate. The gate reassignment is based on the gate reassignment scheduled time. The time node is decided by the acquired information and operation condition the same day. If the delay time of delay flight is confirmed, the arrival-departure time is conformed, so the reassignment demand is urgent.

2.2. Stochastic Factors and Relax Constrains. Influenced by delay features, different flights have different uncertain degrees. Stochastic factors have a significant effect on unidentified flights, which will lead to varieties of uncertain situation. Assume that there are only 2 gates (Gate 1 and Gate 2) and 3 flights (Flight 1, Flight 2, and Flight 3), Figure 1 shows the scheduled gate assignment for 3 flights. 


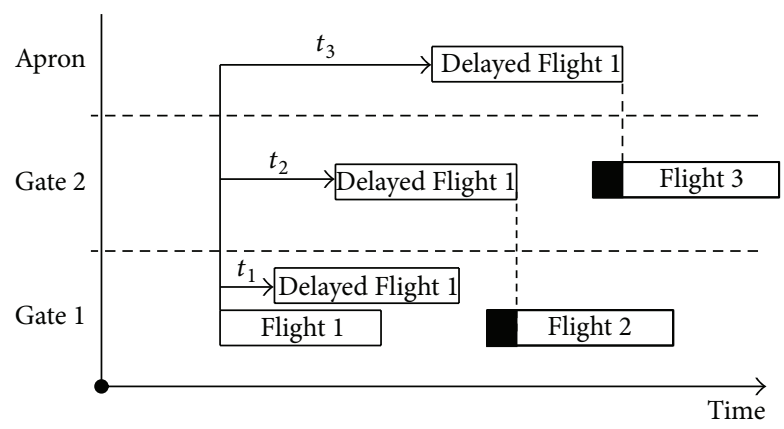

FIGURE 1: Influence on gate reassignment by stochastic factors.

From Figure 1, Flight 1 and Flight 2 are assigned to Gate 1 and Flight 3 is assigned to Gate 2 in the planned assignment stage. The probability of delay time of Flight 1 is one of $t_{1}, t_{2}$, or $t_{3}$. Assume that the probability of each delay scenarios is equal. If the delay time of Flight 1 is $t_{1}$, the planned assignment is still valid. If the delay time of Flight 1 is $t_{2}$, Flight 1 will have time overlap with Flight 2 . To avoid this occasion, Flight 1 can be allocated to Gate 2 or the apron. If the delay time of Flight 1 is $t_{3}$, Flight 1 will have time overlap with Flight 2 and 3 , and it can be only allocated to the apron. When Flight 1 is reassigned to Gate 2, the influence on airport operation cost and service level will be ignored, while when Flight 1 is reassigned to apron, the influence will be worse. At the realtime gate assignment stage, if avoiding occupancy conflict drastically is the main objective in gate reassignment, gate managers should consider all the three delay scenarios and assign Flight 1 to the apron.

From the point of minimizing the reassignment influence, it is just necessary to consider scenarios that delay time are $t_{1}$ and $t_{2}$ if some potential conflicts are acceptable. In this way, Flight 1 can be allocated to Gate 2. Gate managers need to balance the cost and influence caused by potential conflicts with small probabilities.

The influence coming from stochastic factors for uncertain delay information should be taken into consideration. Some constraints of unidentified delay flights are tolerable to be conflicted, which means that time overlap (or time violation) is allowable in one gate. Relax constraints can lead to infeasible solution in some scenarios, which needs penalty coefficients and functions to deal with. Details about penalty coefficients and functions will be described in Section 3. Uncertain flights are not in reassignment urgency, so this violation will not have actual influence on real-time airport operation in an operation framework repeatedly.

2.3. Reassignment Disturbance. There are three main processes for gate reassignment, allocating flights to aprons (apron disturbance), allocating flights to gates different from scheduled (gate disturbance), and making flights wait until gates are available (time disturbance). All of these processes will disturb the normal operation of airport and will have serious impact on passenger service level and stall scheduling. Thus, to maximize airport operation efficiency, benefits, and passenger service level, aforementioned constrains are necessary to be considered. The objective of research is to minimize the reassignment disturbance value and penalty value of certain and uncertain delay flight compared to planned gate assignment. Real-time gate reassignment is a continuous operation process. In order to obtain perfect reassignment scheme, the real-time operation process need a fixed time circle to complete all gate reassignment tasks in one day.

In order to avoid extra delay or propagation delay caused by airport operation for certain flight, only apron disturbance and gate disturbance are considered. On the contrary, for uncertain flight, because they lack reassignment urgency, it is better to delay their gate reassignment starting time rather than assign them to aprons when no gates are available. Thus, gate disturbance, time disturbance, and the penalty caused by relax constraints are considered. In actual airport operation, only the reassignment results of certain flights are released to gate managers, while the calculation results data of uncertain flights are key influence factors to improve the flexibility of certain flight gate reassignment results.

\section{Real-Time Gate Assignment Model}

3.1. Assumption. The assumptions used for the real-time gate assignment model are listed as follows.

(i) The result of planned gate assignment is known in advance.

(ii) The arrival-departure time distribution of each flight is known.

(iii) For simplicity, the research divides the minimum safety interval time and necessary buffer time between two consecutive flights into two parts and pluses them into gate occupation time of the two flights, respectively, according to aircraft type request. Thus, the minimum interval constrains need not to be considered.

(iv) For simplicity and maximum reassignment efficiency, constrains, such as airlines preference, are not considered.

(v) The aprons in airport, which includes three types, are sufficient. When a flight is assigned to the apron, it does not need to consider time overlap and aircraft type matching.

\subsection{Mathematical Notation}

$v_{i, k+1}$ : The disturbance value, Flight $i$ is assigned to apron

$w_{i k}$ : The disturbance value, Flight $i$ is assigned to gate $k$

$C_{i}$ : The gate occupying time point of Flight $i$

$T_{i}$ : The gate occupation time of Flight $i$, including minimum safety interval time and necessary buffer time

$P_{\alpha}$ : Probability of the ath stochastic scenario 
$d_{i j}$ : The delay disturbance value, the uncertain Flight $i$ is assigned to gate at time point $j$

$e_{k t}$ : The penalty value, gate conflict is emerged in the $k$ gate at time point $t$ for uncertain flight

MF: Set of certain flights in each gate reassignment stage

NF: Set of uncertain flights in each gate reassignment stage

$\mathrm{VG}_{i}$ : Set of all available gates assigned to certain Flight $i$

$\mathrm{DG}_{i}$ : Set of all available gates assigned to uncertain Flight $i$

$\mathrm{TD}_{i}$ : Set of available time points assigned to uncertain Flight $i$

$k$ : Set of gates, including several gates

$k+1$ : Set of apron, including several aprons

$M_{i}$ : Aircraft type of Flight $i$

$N_{k}$ : Type of Gate $k$

$B_{i j}^{\alpha}$ : Set of available time point to assign uncertain

Flight $i$ to Gate $k$ at stochastic scenario $a$

$\mathrm{VF}_{j k}$ : Set of certain flights assigned to Gate $k$ at time point $j$

$\mathrm{DF}_{j k}^{\alpha}$ : Set of uncertain flight assigned to Gate $k$ at time point $j$ at stochastic scenario $a$

$H_{S}$ : Set of all available time point to assign uncertain flights

$K_{t}$ : Set of all available gates at time point $t$

$H$ : A sufficient large positive number to avoid time overlap in gate exclusive constraint

$A$ : The number of all stochastic scenarios.

\section{Consider}

$x_{i, k+1}= \begin{cases}1, & \text { certain Flight } i \text { assigned to apron } \\ 0, & \text { otherwise, }\end{cases}$

$x_{i k}= \begin{cases}1, & \text { certain Flight } i \text { assigned to gate } k \\ 0, & \text { otherwise, }\end{cases}$

$x_{i j k}= \begin{cases}1, & \text { certain Flight } i \text { assigned to Gate } k \text { at time } j \\ 0, & \text { otherwise, }\end{cases}$

$y_{i j k}= \begin{cases}1, & \text { uncertain Flight } i \text { assigned to gate } k \text { at time } j \\ 0, & \text { otherwise, }\end{cases}$

$h_{k t}^{\alpha}= \begin{cases}1, & \text { at stochastic scenario } a, \\ & \text { time violation occurs in gate } k \text { at time } j \\ 0, & \text { otherwise. }\end{cases}$
3.3. Objective Function. The basic ideology of the objective function in the paper deems that the original gate assignment is the optimal scheme under no flight delay. The objective function is consisting of three parts. The first part is the gate reassignment aimed at the certain information; the second part is the gate reassignment aimed at the uncertain information; the third part is to analyze the influence of gate assignment under certain information on the gate assignment under uncertain information. The objective function selected in the paper is to minimize the cost of gate assignment, which is caused by flight delay, rather than act the arrival-departure time as the input to gate reassignment. Therefore, the gate assignment schedule is not the optimal schedule aiming at some gates but the schedule ensuring the minimum number of flight delay accord with the basic principle of airport operation and management. The optimal schedule of gate assignment has not an effect on the normal order of airport operation and cannot lead to safety risk.

The First Part. Reassignment of certain flight is as follows:

$$
\min Z_{1}=\sum_{i \in \mathrm{MF}} V_{i, k+1} x_{i, k+1}+\sum_{i \in \mathrm{MF}} \sum_{k \in \mathrm{VG}_{i}} w_{i k} x_{i k}
$$

Objective function (2) aims to minimize apron disturbance value and gate disturbance value of certain flights gate reassignment. Assuming that gate number is $m$, space interval between reassigned gates and planned gates is $n$. To reduce the number of changed gates and the influence on additional distance of passengers and staffs, the authors set gate disturbance value as $m+n$. Due to larger influence on passenger service level and airport operation efficiency caused by reassigning the flights to apron, apron disturbance value is $2 \mathrm{~m}$.

Part Two. Reassignment of uncertain flight is as follows:

$$
\begin{aligned}
& \min Z_{2} \\
& =\sum_{\alpha=1}^{A} P_{\alpha}\left[\sum_{i \in \mathrm{NF}} \sum_{j=B_{j k}^{1} \cup B_{j k}^{2} \cdots B_{j k}^{A}} \sum_{k \in \mathrm{DG}_{i}}\left(w_{i k}+d_{i j}\right) y_{i j k}\right. \\
& \left.\quad+\sum_{t \in T} \sum_{k \in K_{t}} e_{k t} h_{k t}^{a}\right] .
\end{aligned}
$$

Objective function (3) aims to minimize gate disturbance value, time disturbance value, and relax constraint penalty value of uncertain flights gate reassignment. Gate disturbance value is the same as in function (2). Assume that gate number is $m$, waiting time is $t$, considering influence on passenger service level and airline fuel consumption by delay gate starting time, and time disturbance coefficient is $(t / 10) m$. To 
minimize the infeasible situation caused by relax constraints under stochastic factors, penalty coefficient is $(4 t / 5) \mathrm{m}$.

Part Three. Penalty functions of certain flights and uncertain flights are as follows:

$\min Z_{3}$

$=\sum_{\alpha=1}^{A} P_{\alpha}\left\{\sum_{i \in \mathrm{NF} \cup \mathrm{MF}} \sum_{j=B_{j k}^{1} \cup B_{j k}^{2} \cdots B_{j k}^{A}} \sum_{k \in \mathrm{VG}_{i} \cup \mathrm{DG}_{i}} e_{k t}\left[1-\left(x_{i j k}-y_{i j k}\right)^{2}\right]\right\}$.

Penalty function (4) is designed to minimize the violation, by which the reassignment of certain flights may have disturbance on planned assignment of uncertain flights. The penalty value of the violation is the same as the relax constraint penalty value in function (3). The integrated objective function is the sum of all the three abovementioned functions:

$$
\min Z=Z_{1}+Z_{2}+Z_{3}
$$

3.4. Constraints. Consider the following:

$$
\begin{gathered}
\sum_{k \in \mathrm{VG}_{i} \cup k+1} x_{i k}=1, \quad \forall i \in \mathrm{MF}, \\
x_{i k}=\left\{\begin{array}{l}
1 \\
0,
\end{array} \quad \forall i \in \mathrm{MF}, \quad \forall k \in \mathrm{VG}_{i} \cup k+1 .\right.
\end{gathered}
$$

Constraint (6) is flight unique constraint. It indicates that each certain flight must be assigned to only one gate or assigned to apron. Constraint (7) indicates the certain flight variables are either zero or one:

$$
\begin{gathered}
\sum_{j=B_{i k}^{\alpha}} \sum_{k \in \mathrm{DG}_{i}} y_{i j k}=1, \quad \forall i \in \mathrm{NF}, \\
y_{i j k}=\left\{\begin{array}{l}
1 \\
0,
\end{array} \quad \forall i \in \mathrm{NF}, \forall j \in B_{i k}^{1} \cup B_{i k}^{2} \cdots \cup B_{i k}^{A}, \forall k \in \mathrm{DG}_{i} .\right.
\end{gathered}
$$

Constraint (8) is flight unique constraint. It indicates that each uncertain flight must be assigned to only one gate at each stochastic scenario. Constraint (9) indicates that the uncertain flights variables are either zero or one:

$$
\begin{gathered}
\sum_{j=B_{i k}^{\alpha}} \sum_{k \in \mathrm{VG}_{i}} x_{i j k}=1, \quad \forall i \in \mathrm{MF}, \\
x_{i j k}=\left\{\begin{array}{l}
1 \\
0,
\end{array} \quad \forall i \in \mathrm{MF}, \forall j \in B_{i k}^{1} \cup B_{i k}^{2} \cdots \cup B_{i k}^{A}, \forall k \in \mathrm{VG}_{i} .\right.
\end{gathered}
$$

Constraint (10) is flight unique constraint. It indicates that, in penalty function, each certain flight must be assigned to only one gate at each stochastic scenario. Constraint (11) indicates that the certain flights values are either zero or one:

$$
\begin{gathered}
C_{i^{\prime}} \geq C_{i}+T_{i}+H\left(x_{i^{\prime} k}+x_{i k}-2\right), \\
i \leq i^{\prime}, \quad \forall i \in \mathrm{MF}, \quad \forall i^{\prime} \in \mathrm{MF}, \quad \forall k \in \mathrm{VG}_{i} .
\end{gathered}
$$

Constraint (11) is gate exclusive constraint. It indicates that, at one period of time, one gate can only serve less than or equal one certain flight. In the same gate, the gate starting time point of subsequent flight $i^{\prime}$ should be greater than the gate starting time point plus gate occupation time of front flight $i$. $H\left(x_{i^{\prime} k}+x_{i k}-2\right)$ is used to check if Flights $i$ and $i^{\prime}$ are reassigned to the same gate:

$$
\begin{gathered}
\sum_{j \in H_{s}} \sum_{i \in \mathrm{VF}_{j k}} y_{i j k}-h_{k t}^{\alpha} \leq 1, \quad \forall k \in K_{t}, \quad \forall t \in T, \\
h_{k t}^{\alpha} \geq 0, \quad \forall k \in K_{t}, \quad \forall t \in T .
\end{gathered}
$$

Constraint (13) is the relax gate exclusive constraint. It indicates that at one period of time, more than one uncertain flight are tolerable to be assigned to one gate. In one gate, the flight number minus violation number must be less than or equal to one. Constraint (14) is to ensure that the violation number is a positive number:

$$
M_{i} \cdot x_{i k} \leq N_{k}, \quad \forall k \in \mathrm{VG}_{i} .
$$

Constraint (15) is aircraft type matching constraint. The aircraft type of certain flights should be less than or equal to gate type:

$$
M_{i} \cdot y_{i j k} \leq N_{k}, \quad \forall k \in \mathrm{DG}_{i}, \quad \forall i \in \mathrm{NF}, \forall j \in B_{i k}^{1} \cup B_{i k}^{2} \cdots B_{i k}^{A} .
$$

Constraint (16) is aircraft type matching constraint. The aircraft type of uncertain flights should be less than or equal to gate type.

\section{Ant-Based Heuristic Algorithms}

4.1. Algorithm Selection. The paper selects Ant colony algorithm to solve the gate assignment problem. Ant colony optimization algorithm is a metaheuristic optimization method proposed by Dorigo et al. [16]. Dorigo presented the ant colony algorithm for the first time in 1992. The ant colony algorithm has the characteristic of solving the discrete combinatorial optimization problem. The typical combinatorial optimization problem includes: traveling salesman problem (TSP) $[17,18]$, machine scheduling problem (MSP) $[19,20]$, vehicle routing problem (VRP) $[21,22]$, and parallel machine scheduling (PMS) [23, 24].

There are some reasons to explain the selection of ant colony algorithm. First, ant colony algorithm is applied to solve combinatorial optimization problem, which accords with the characteristics of gate assignment. Second, the characteristics of ant colony algorithm are to add the solution to the solution system step by step until it acquires a complete solution. Therefore, it is superior to solve gate assignment relative to adjusting the part solution of algorithm.

4.2. Description of Ant Colony Algorithm. Our research designs an ant colony algorithm to solve the gate reassignment problem. The set of the number of ants is $k$, and the ant indicates the considered flight alignment. Ants firstly 


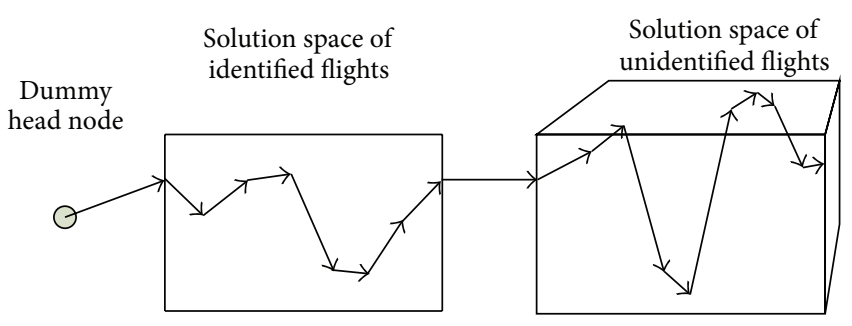

FIGURE 2: Integrated searching of ants.

walk through solution space of identified flights, then walk from one of the unidentified flights. After traversal of the two spaces, each ant releases suitable pheromone on each node passed, according to the target value of ant path. In reassignment process of identified flight, the space, which is a dimensional grid $m \times n$, consists of gate (apron) and flight alignment, and each node in grid indicates a possible result which is meant to assign a flight to a gate. Ants start form a dummy head node $F_{0}$, choose a node from the following row based on pheromone and heuristic information of each node, and then repeat until reach the last row. If a node cannot satisfy the constraints, ants will ignore this node. In reassignment process of unidentified flight, the space is a threedimensional grid $m \times n \times t$ which consists of gate (apron), flight alignment, and available time point; each node in grid indicates a possible result which is meant to assign a flight to a gate at a time point. In unidentified flight solution space, the ants' searching method is similar to that of identified flight. Each time ants finish the above-mentioned stage, the ants will be deleted, and then a second iterative process will start. Figure 2 shows the ants' traversal process.

4.2.1. Heuristic Information Setting. Setting heuristic information as $\eta_{i}(t)$ means that the attractiveness of node $i$ is at time $t$ :

$$
\eta_{s}(t)= \begin{cases}a, & a=P_{\text {assigned }} \cup(P, Q)_{\text {assigned }}, a>b \\ b, & \text { otherwise, }\end{cases}
$$

where $P_{\text {assigned }}$ indicates sets of planned assignment gate alignment. $(P, Q)_{\text {assigned }}$ indicates sets of planned assignment gate and time point alignment. Gate assignment has a greater attractiveness to ants in order to enhance the probability of selecting scheduled gates.

4.2.2. State Transition Equation. When ants $k(k=1,2, \ldots$, $m$ ) search nodes, its state transition probability is based on the pheromone concentration and heuristic information of the following nodes. $p_{i}^{k}(t)$ indicates the state transition probability of ant $k$ transferring from its located node into node $i$ at time $t$. The expression is shown as follows:

$$
p_{i}^{k}(t)=\left\{\begin{array}{l}
\frac{\left[\tau_{i}(t)\right]^{\alpha} \cdot\left[\eta_{i}(t)\right]^{\beta}}{\sum_{s \in \text { allowed }}\left[\tau_{s}(t)\right]^{\alpha} \cdot\left[\eta_{s}(t)\right]^{\beta}}, \\
0,
\end{array}\right.
$$

where $\tau_{i}(t)$ indicates the pheromone concentration of node $i$ at time point $t$ and allowed ${ }_{k}$ indicates the available nodes for ant $k$ to choose in the next stage, which satisfies the constraints of gate reassignment problem. Elements in set allowed $_{k}$ may change according to the choice of ant $k$. Ants must select nodes in the following row or space in identified flights grid or unidentified flights grid, respectively, so reduplicative searching is avoided. Factors $\alpha$ and $\beta$ determine the relative importance of pheromone accumulated on nodes when it has an impact on choice of ants.

4.2.3. Pheromone Update Methods. When ants complete an iteration, pheromone on each node should be updated. New pheromone will be added to nodes, while residual pheromone on each node should be volatilized. Therefore, the rule of pheromone modulation is shown as follows:

$$
\begin{gathered}
\tau_{i}(t+1)=\rho \tau_{i}(t)+\Delta \tau_{i}^{\text {best }}, \\
\Delta \tau_{i}= \begin{cases}\frac{Q}{f\left(k^{\text {best }}\right)}, & i \in k^{\text {best }} \\
0, & i \notin k^{\text {best }},\end{cases}
\end{gathered}
$$

where $\rho$ indicates volatile coefficient of pheromone, $Q$ indicates pheromone strength, and $\Delta \tau_{i}$ indicates the total pheromone increment on node $i$ at present iteration. Only the optimal ants release pheromone at each iteration.

\subsection{Algorithm Flow. Figure 3 shows the algorithm flow.}

The model can select PSO method to complete the relative parameters learning in ant colony algorithm in order to avoid the defects of ant colony algorithm itself under large-scale flight delay.

\section{Simulation and Verification}

In the numerical test, the starting and ending time of certain flights gate assignment are determined by two parts: one is the latest estimated time of departure (ETD) and arrival (ETA) delivered by airlines; the other is the minimum safety interval time and necessary buffer time according to aircraft types. The time of uncertain flights is also determined by the two parts as Table 1 shows; the difference between them is that certain flights utilize the scheduled time of depart (STD) and arrival (STA). In Table 1, the bold font represents the actual time of departure and arrival for the flight. The gate reassignment is based on the present gate occupation situation and sequencing of flights confirmed by updated gate starting time. Each time the gate reassignment aims at all flights, while only the reassignment scheme of identified flights can be released.

The simulation and verification in the paper is based on operation of a hub airport in China. The airport has a passenger throughput of more than 19 million and aircraft movements of 166 thousand, which is an important regional hub airport of East China. The Terminal B in the airport is domestic terminal, which has 41 gates and consists of 6 gates of type $\mathrm{E}, 7$ gates of type $\mathrm{D}$, and 28 gates of type $\mathrm{C}$. In the simulation and verification, we assume that the gates and aprons have enough capacity such as sufficient runway and taxiway systems. Data is based on the timetable of May 20, 
TABLE 1: Values of gate stating and ending time.

\begin{tabular}{lccccc}
\hline Flight type & Flight number & STA & ETA & STD & ETD \\
\hline \multirow{2}{*}{ Identified flight } & $\mathrm{f} 1$ & 1035 & $\mathbf{1 1 1 0}$ & 1140 & $\mathbf{1 3 0 5}$ \\
& $\mathrm{f} 2$ & 1125 & $\mathbf{1 1 2 2}$ & 1225 & $\mathbf{1 2 3 6}$ \\
\hline \multirow{2}{*}{ Unidentified flight } & $\mathrm{f} 3$ & $\mathbf{1 1 5 0}$ & $\mathbf{-}$ & $\mathbf{1 2 4 5}$ & $\mathbf{1 3 0 0}$ \\
& $\mathrm{f} 4$ & $\mathbf{1 1 5 5}$ & - \\
\hline
\end{tabular}

The bold font represents the actual time of departure and arrival for the flight.

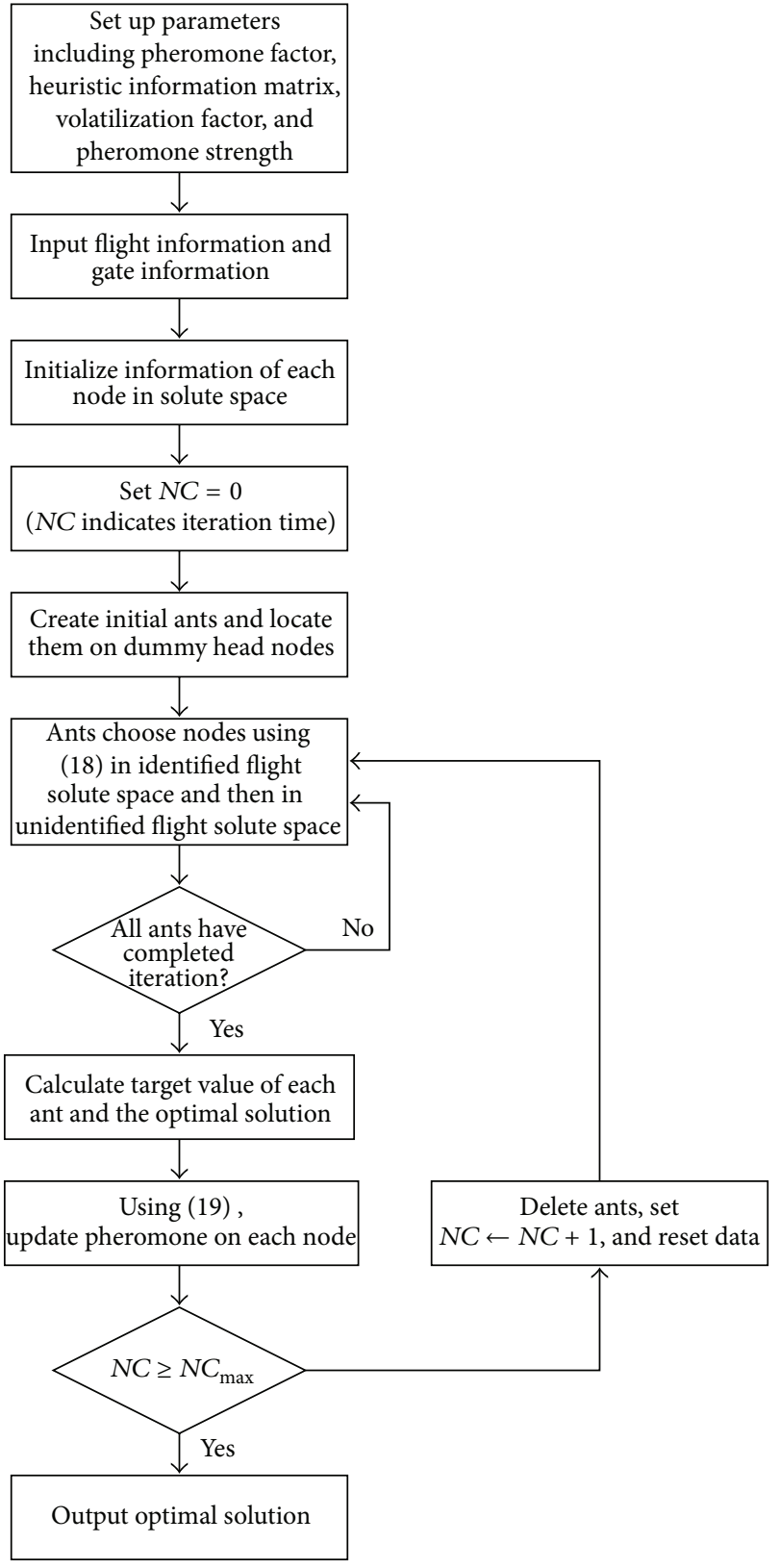

FIgURE 3: Algorithm flow.

2013, of Terminal B, when there are 418 aircraft movements or 281 flight pairs, including departure, transferring, and arrival flights. The evaluation is based on the planned gate assignment of flights and the real-time tracked data between 6:00 and 15:00 which is used to be input information to gate

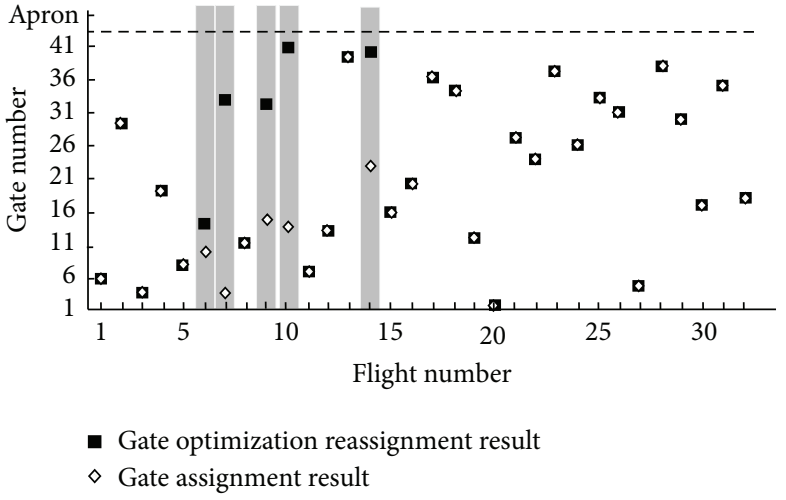

FIGURE 4: Gate optimization reassignment result at time point 9:00.

reassignment. Affected by storm at that day, extensive flights delayed at about 12:00, and there was considerable deviation between flights schedule and real-time operation at airport.

This research selects time points 7:00, 9:00, 11:00, 13:00, and 15:00 to perform the test and has a comparison with manual reassignment methods. The manual method which based on experience operates can be used as follows: if there is a free gate when conflict occurs, assign the flight to the free gate, otherwise, assign to apron. The test is performed on an AMD TurionX2 $2.2 \mathrm{GHz}$ with $2 \mathrm{~GB}$ RAM in the environment of Microsoft Windows Vista and uses the C computer language to write the program.

Based on the case of gate reassignment of time point 9:00, the number of considered flights which needs to be allocated is 246, which includes 32 identified flights. Table 2 shows flight number, planned assignment result, manual reassignment result, and optimization reassignment result of identified flights at this time point.

Gate optimization and manual reassignment results are shown in Figures 4 and 5.

Figure 3 shows the test result of time point 9:00. At that time, 246 flights need to be reassigned, including 32 identified flights, which should display the specific reassignment result.

As shown in Figure 3, only Flights 6, 7, 9, 10, and 14 need to be reassigned, others keep their gates, and none of them are assigned to the apron. As shown in Figure 4, Flights 4, 7, $9,10,14,16$, and 29 need to be reassigned, and none of Flights 10,14 , and 16 are allocated to aprons. It is shown that gate optimization reassignment result causes less disturbances to aircraft, and the total disturbance value of identified flights and unidentified flights is 1535.642 . The disturbance value of identified flights in optimization result is 1314.537 while being 1683 in manual result. Therefore, in actual situation, test 
TABLE 2: Gate reassignment results of identified flight at time point 9:00.

\begin{tabular}{|c|c|c|c|c|c|}
\hline $\begin{array}{l}\text { Flight } \\
\text { number }\end{array}$ & ETA & ETD & $\begin{array}{c}\text { Planned } \\
\text { assign- } \\
\text { ment result }\end{array}$ & $\begin{array}{c}\text { Manual } \\
\text { reassign- } \\
\text { ment result }\end{array}$ & $\begin{array}{l}\text { Optimized } \\
\text { reassign- } \\
\text { ment result }\end{array}$ \\
\hline 1 & 900 & 1044 & 6 & 6 & 6 \\
\hline 2 & 906 & 1025 & 29 & 29 & 29 \\
\hline 3 & 911 & 1025 & 3 & 3 & 3 \\
\hline 4 & 915 & 1106 & 19 & 19 & 19 \\
\hline 5 & 924 & 1048 & 8 & 14 & 8 \\
\hline 6 & 925 & 1113 & 10 & 10 & 14 \\
\hline 7 & 929 & 1103 & 4 & 20 & 33 \\
\hline 8 & 942 & 1109 & 11 & 11 & 11 \\
\hline 9 & 945 & 1127 & 15 & 30 & 32 \\
\hline 10 & 948 & 1154 & 14 & Apron & 41 \\
\hline 11 & 954 & 1127 & 7 & 7 & 7 \\
\hline 12 & 955 & 1105 & 13 & 13 & 13 \\
\hline 13 & 956 & 1133 & 39 & 39 & 39 \\
\hline 14 & 956 & 1130 & 23 & Apron & 40 \\
\hline 15 & 1000 & 1100 & 16 & 16 & 16 \\
\hline 16 & 1006 & 1136 & 20 & Apron & 20 \\
\hline 17 & 1010 & 1050 & 36 & 36 & 36 \\
\hline 18 & 1012 & 1310 & 34 & 34 & 34 \\
\hline 19 & 1015 & 1136 & 12 & 12 & 12 \\
\hline 20 & 1030 & 1200 & 2 & 2 & 2 \\
\hline 21 & 1030 & 1155 & 27 & 27 & 27 \\
\hline 22 & 1031 & 1208 & 24 & 24 & 24 \\
\hline 23 & 1034 & 1200 & 37 & 37 & 37 \\
\hline 24 & 1037 & 1318 & 26 & 26 & 26 \\
\hline 25 & 1039 & 1148 & 33 & 33 & 33 \\
\hline 26 & 1042 & 1227 & 31 & 31 & 31 \\
\hline 27 & 1045 & 1220 & 5 & 5 & 5 \\
\hline 28 & 1045 & 1213 & 38 & 38 & 38 \\
\hline 29 & 1048 & 1217 & 30 & 25 & 30 \\
\hline 30 & 1050 & 1210 & 17 & 17 & 17 \\
\hline 31 & 1051 & 1213 & 35 & 35 & 35 \\
\hline 32 & 1059 & 1156 & 18 & 18 & 18 \\
\hline
\end{tabular}

results of optimization reassignment in our research have an obvious advantage over manual reassignment. In the test, the relative time we set for valid time of identified flights is long, so the influence of unidentified flights is low. The influence proportion of unidentified flights is adjustable according to real-time situation.

Table 3 and Figures 6 and 7 present the comparison results of optimization reassignment and manual reassignment of the above-mentioned 5 time points.

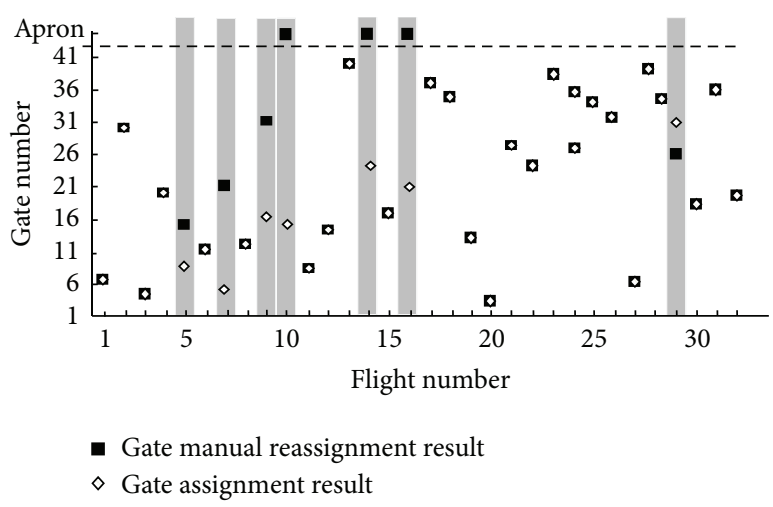

Figure 5: Gate manual reassignment result at time point 9:00.

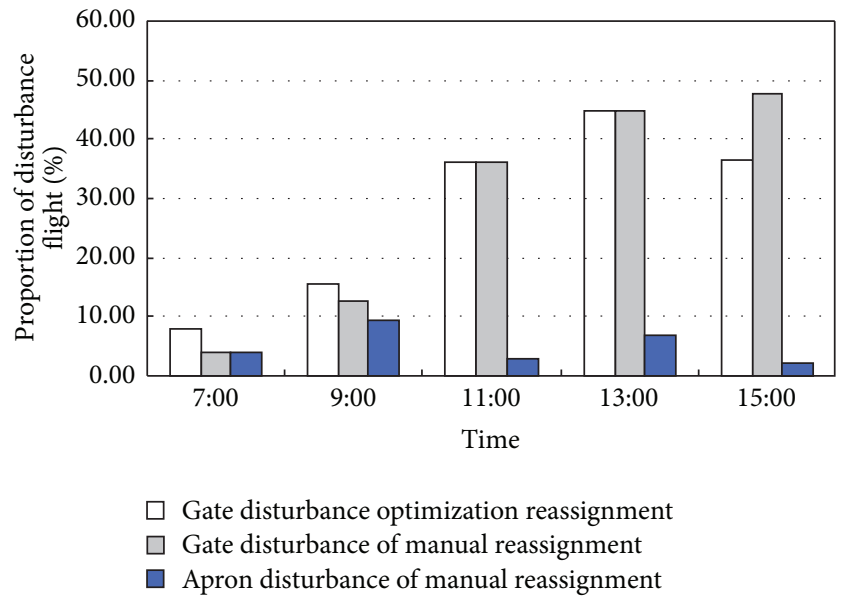

FIGURE 6: Comparisons of optimization and manual gate reassignment on disturbance proportion.

As shown in Figures 6 and 7, in operation day, the quantity of irregular flights largely increases, which leads to the more complex gate reassignment. Before time point 12:00, the situation in airport is relatively normal. The proportion of fights gate reassignment is less than $20 \%$ and disturbance value is small. After extensive delays happen, the disturbance value of reassignment largely increases. Although optimization result has considerable deviation compared to the planned gate assignment, the efficiency and systematic optimization are much better than manual reassignment. The proportion of disturbed flights in optimization result is less than $45 \%$, while that of manual results is more than $50 \%$ with a larger disturbance value.

In the aforementioned tests of 5 time point, none of the flights are allocated to the aprons. Proportion and disturbance value of disturbed identified flights in optimization results are less than those of manual reassignment results. The maximum decrement of disturbed flights proportion reaches $13.64 \%$ at $15: 00$, while the maximum decrement of disturbance value is 327.093 at 9:00. As a consequence, the optimization gate reassignment strategy is better than the manuals, no matter in one operation or one day's operation. The resolving time for performing tests by ant-based 
TABLE 3: Comparisons of optimization and manual gate reassignment.

\begin{tabular}{|c|c|c|c|c|c|c|c|c|}
\hline $\begin{array}{l}\text { Time } \\
\text { point }\end{array}$ & $\begin{array}{l}\text { Reassignment } \\
\text { method }\end{array}$ & $\begin{array}{c}\text { Gate } \\
\text { disturbance } \\
\text { proportion }\end{array}$ & $\begin{array}{c}\text { Apron } \\
\text { disturbance } \\
\text { proportion }\end{array}$ & $\begin{array}{c}\text { Total } \\
\text { disturbance } \\
\text { proportion }\end{array}$ & $\begin{array}{l}\text { Identified } \\
\text { flight number }\end{array}$ & $\begin{array}{c}\text { Total } \\
\text { considered } \\
\text { flight number }\end{array}$ & $\begin{array}{c}\text { Disturbance } \\
\text { value }\end{array}$ & Time costs (s) \\
\hline \multirow{2}{*}{$6: 00$} & Optimization & $8 \%$ & 0 & $8 \%$ & 50 & 281 & 2051.073 & \multirow{2}{*}{184.746} \\
\hline & Manual & $4 \%$ & $4 \%$ & $8 \%$ & 50 & 281 & 2173.52 & \\
\hline \multirow{2}{*}{ 9:00 } & Optimization & $15.60 \%$ & 0 & $15.60 \%$ & 32 & 246 & 1314.537 & \multirow{2}{*}{117.996} \\
\hline & Manual & $12.50 \%$ & $9.40 \%$ & $21.90 \%$ & 32 & 246 & 1686.63 & \\
\hline \multirow{2}{*}{ 11:00 } & Optimization & $36.11 \%$ & 0 & $36.11 \%$ & 36 & 207 & 1480.92 & \multirow{2}{*}{78.622} \\
\hline & Manual & $36.11 \%$ & $2.78 \%$ & $38.89 \%$ & 36 & 207 & 1604.34 & \\
\hline \multirow{2}{*}{ 13:00 } & Optimization & $44.80 \%$ & 0 & $44.80 \%$ & 29 & 173 & 1028.72 & \multirow{2}{*}{77.729} \\
\hline & Manual & $44.80 \%$ & $6.90 \%$ & $51.70 \%$ & 29 & 173 & 1317.35 & \\
\hline \multirow{2}{*}{ 15:00 } & Optimization & $36.36 \%$ & 0 & $36.36 \%$ & 44 & 151 & 1698.24 & \multirow{2}{*}{105.112} \\
\hline & Manual & $47.73 \%$ & $2.27 \%$ & $50.00 \%$ & 44 & 151 & 1931.66 & \\
\hline
\end{tabular}

algorithm has a tendency to decrease with the decrease of considered flight. The longest resolving time is $184.746 \mathrm{~s}$, which can reach the strict request of real-time operation.

Gate manual reassignment only considers recent flight information and actual gate utilization situation, while optimization reassignment can consider the long time in some degrees. Optimization reassignment will follow the airport delay trending order to look for more satisfied reassignment schemes. Therefore, it will largely decline the disturbance of gate reassignment.

Thus it can be seen that, in actual situation, test results of optimization reassignment in our research are much better than those in manual reassignment. The similarity between optimization gate reassignment and planned gate assignment is high.

\section{Conclusions}

In the paper, airport gate reassignment problem under flight delay situation is studied. Flights are divided into different types considering delay feature. Real-time gate reassignment model has been presented, whose objective is to minimize the disturbance compared to planned gate assignment scheme. An ant-based heuristic algorithm is designed to solve the problem. With the numerical test, the method is proved to be effective and efficient, which can meet the request of realtime operation of hub airport.

Some conclusions can be drawn in the paper. First, the real-time gate assignment is very important in increasing the effectiveness and volume of airport. Second, the real-time gate assignment is difficult because that the flight delay is uncertain. Last, the gate reassignment schedule is decided by the selection of objective function directly. Airport operation and some kinds of influence factors should be taken into account in the actual gate assignment schedule. Therefore, the gate assignment is a typical a multiobjective optimization problem. It should be noted that the research only considers the general situation of gate reassignment. How to operate gate reassignment considering the tradition of gate assignment and importance of flight priority and the reassignment

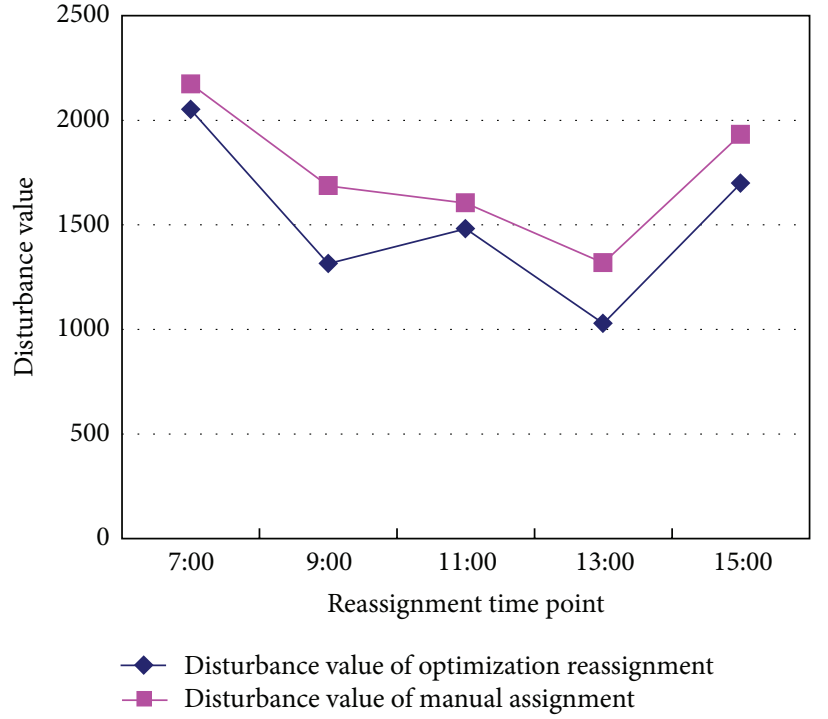

FIGURE 7: Comparisons of optimization and manual reassignment on disturbance proportion.

after situation as airport closures is worthy future research topics.

\section{Acknowledgments}

This work was supported by National Natural Science Foundation and Aviation Fund of China U1233115, Civil Aviation Science Fund of China (MHRD201127), and Qinglan Project.

\section{References}

[1] S. Yan and C.-M. Chang, "A network model for gate assignment," Journal of Advanced Transportation, vol. 32, no. 2, pp. 176-189, 1998.

[2] B. Maharjan and T. I. Matis, "Multi-commodity flow network model of the flight gate assignment problem," Computers \& Industrial Engineering, vol. 63, no. 4, pp. 1135-1144, 2012. 
[3] H. M. Genç, O. K. Erol, B. Eksin, M. F. Berber, and B. O. Güleryüz, "A stochastic neighborhood search approach for airport gate assignment problem," Expert Systems with Applications, vol. 39, no. 1, pp. 316-327, 2012.

[4] C. H. Cheng, S. C. Ho, and C. L. Kwan, "The use of meta-heuristics for airport gate assignment," Expert Systems with Application, vol. 39, no. 16, pp. 12430-12437, 2012.

[5] U. Dorndorf, F. Jaehn, and E. Pesch, "Modelling robust flightgate scheduling as a clique partitioning problem," Transportation Science, vol. 42, no. 3, pp. 292-301, 2008.

[6] C. H. Tang and W. C. Wang, "Airport gate assignment for airline-specific gates," Journal of Air Transport Management, vol. 30, pp. 10-16, 2013.

[7] F. Jaehn, "Solving the flight gate assignment problem using dynamic programming," Zeitschrift für Betriebswirtschaft, vol. 80, no. 10, pp. 1027-1039, 2010.

[8] B. Maharjan and T. I. Matis, "An optimization model for gate reassignment in response to flight delays," Journal of Air Transport Management, vol. 17, no. 4, pp. 256-261, 2011.

[9] Y. Gu and C. A. Chung, "Genetic algorithm approach to aircraft gate reassignment problem," Journal of Transportation Engineering, vol. 125, no. 5, pp. 384-389, 1999.

[10] Y. Z. Hou, An Approach for Dynamic Airport Gate Assignments for Stochastic Flight Delays, National Central University, Jhongli, Taoyuan, 2007.

[11] C.-H. Tang, S. Yan, and Y.-Z. Hou, "A gate reassignment framework for real time flight delays," 4OR, vol. 8, no. 3, pp. 299-318, 2010.

[12] C.-H. Tang, "A gate reassignment model for the taiwan taoyuan airport under temporary gate shortages and stochastic flight delays," IEEE Transactions on Systems, Man, and Cybernetics A, vol. 41, no. 4, pp. 637-650, 2011.

[13] S. Yan and C.-H. Tang, "A heuristic approach for airport gate assignments for stochastic flight delays," European Journal of Operational Research, vol. 180, no. 2, pp. 547-567, 2007.

[14] M. Şeker and N. Noyan, "Stochastic optimization models for the airport gate assignment problem," Transportation Research E, vol. 48, no. 2, pp. 438-459, 2012.

[15] S. Yan, C.-Y. Shieh, and M. Chen, "A simulation framework for evaluating airport gate assignments," Transportation Research $A$, vol. 36, no. 10, pp. 885-898, 2002.

[16] M. Dorigo, V. Maniezzo, and A. Colorni, "Ant system: optimization by a colony of cooperating agents," IEEE Transactions on Systems, Man, and Cybernetics B, vol. 26, no. 1, pp. 29-41, 1996.

[17] A. Uğur and D. Aydin, "An interactive simulation and analysis software for solving TSP using Ant Colony Optimization algorithms," Advances in Engineering Software, vol. 40, no. 5, pp. 341-349, 2009.

[18] C. García-Martínez, O. Cordón, and F. Herrera, "A taxonomy and an empirical analysis of multiple objective ant colony optimization algorithms for the bi-criteria TSP," European Journal of Operational Research, vol. 180, no. 1, pp. 116-148, 2007.

[19] V. T'kindt, N. Monmarché, F. Tercinet, and D. Laügt, "An Ant Colony Optimization algorithm to solve a 2-machine bicriteria flowshop scheduling problem," European Journal of Operational Research, vol. 142, no. 2, pp. 250-257, 2002.

[20] S. Marimuthu, S. G. Ponnambalam, and N. Jawahar, "Threshold accepting and Ant-colony optimization algorithms for scheduling m-machine flow shops with lot streaming," Journal of Materials Processing Technology, vol. 209, no. 2, pp. 1026-1041, 2009.
[21] Y. Gajpal and P. L. Abad, "Multi-ant colony system (MACS) for a vehicle routing problem with backhauls," European Journal of Operational Research, vol. 196, no. 1, pp. 102-117, 2009.

[22] J. F. Tang, Y. Y. Ma, J. Guan et al., "A Max-Min Ant System for the split delivery weighted vehicle routing problem," Expert Systems with Applications, vol. 40, no. 15, pp. 7468-7477, 2013.

[23] T. Keskinturk, M. B. Yildirim, and M. Barut, "An ant colony optimization algorithm for load balancing in parallel machines with sequence-dependent setup times," Computers and Operations Research, vol. 39, no. 6, pp. 1225-1235, 2012.

[24] J. Behnamian, M. Zandieh, and S. M. T. F. Ghomi, "Parallelmachine scheduling problems with sequence-dependent setup times using an ACO, SA and VNS hybrid algorithm," Expert Systems with Applications, vol. 36, no. 6, pp. 9637-9644, 2009. 


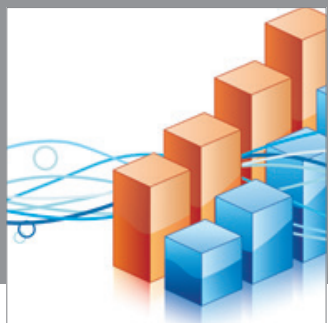

Advances in

Operations Research

mansans

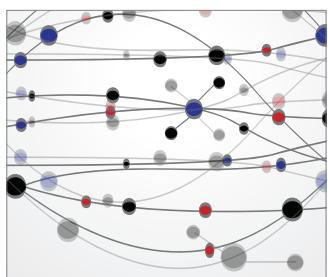

The Scientific World Journal
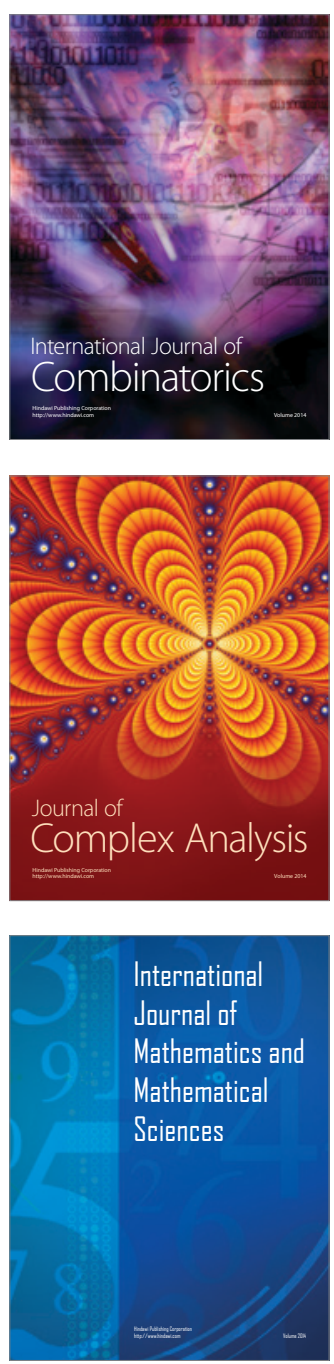
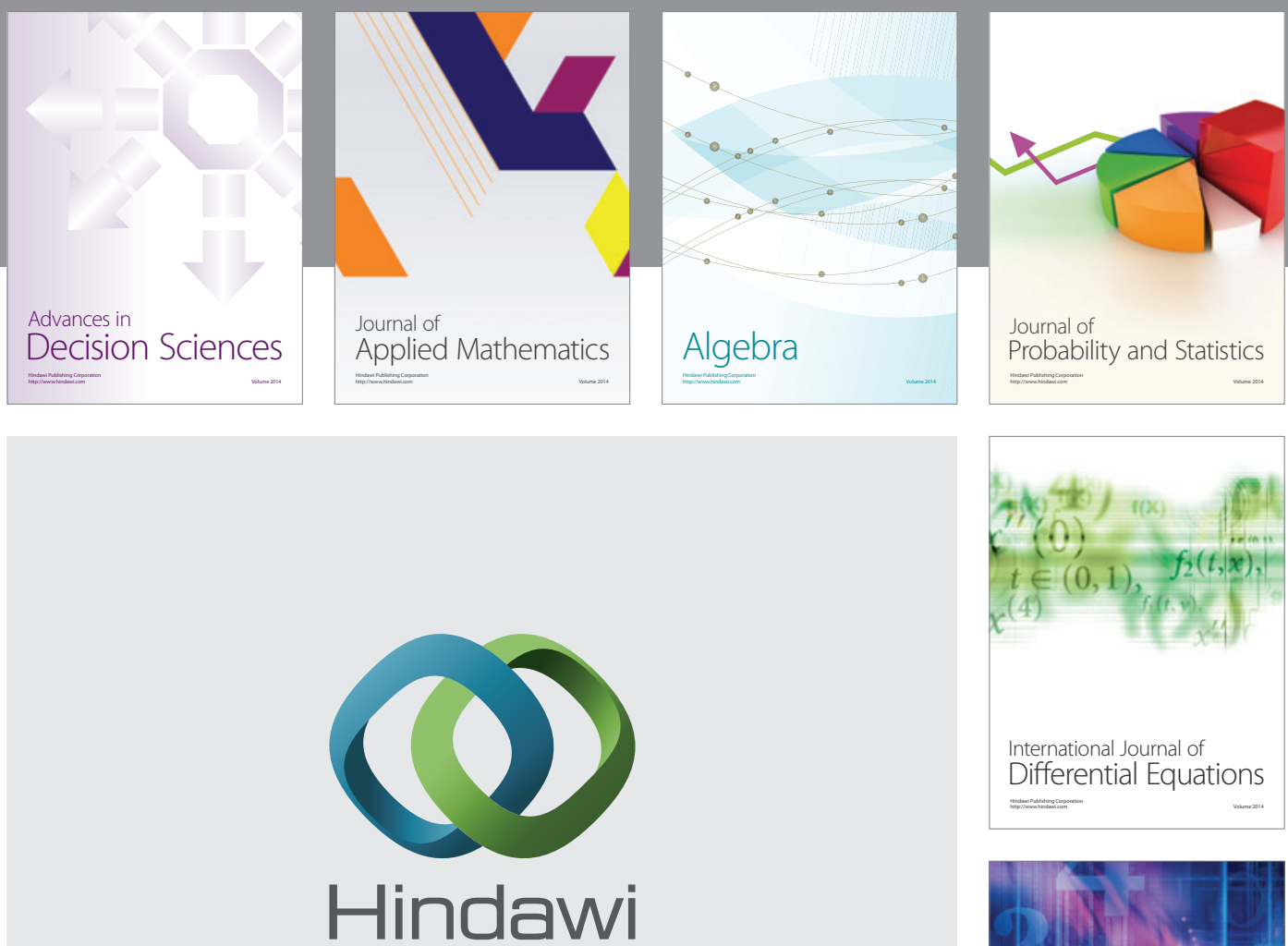

Submit your manuscripts at http://www.hindawi.com
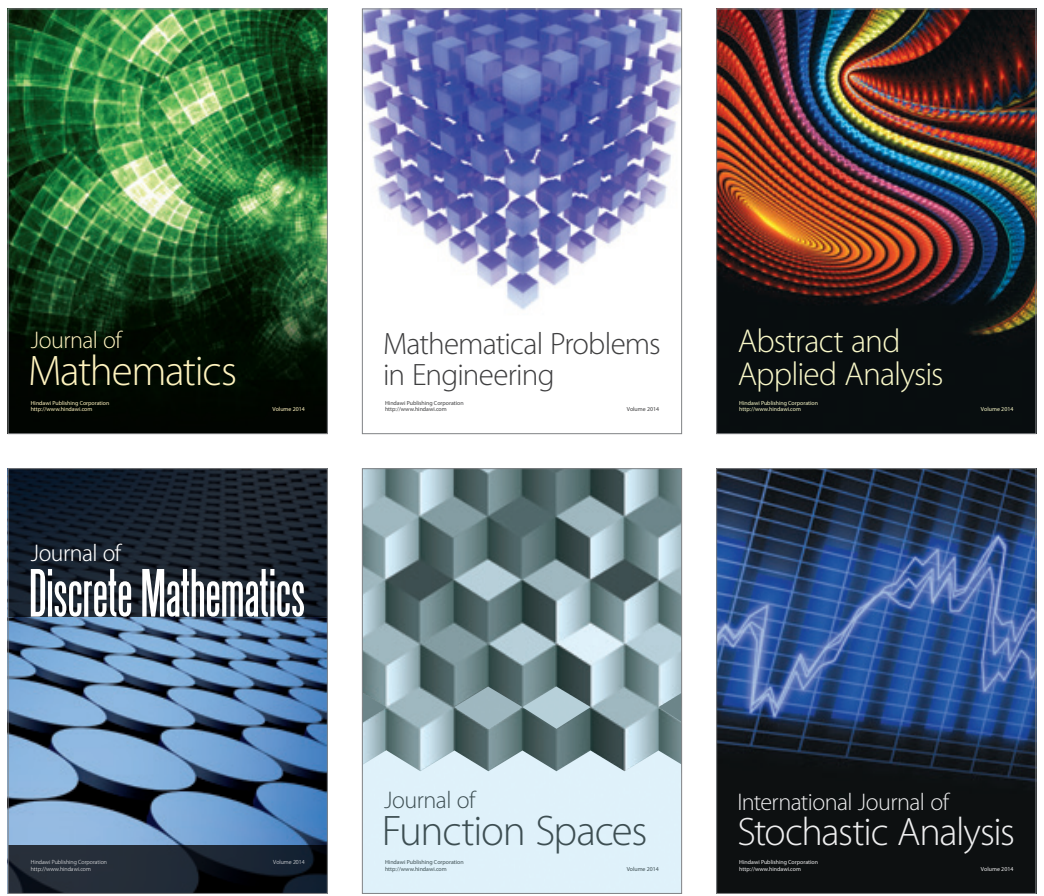

Journal of

Function Spaces

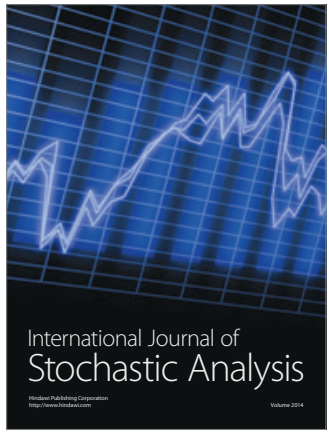

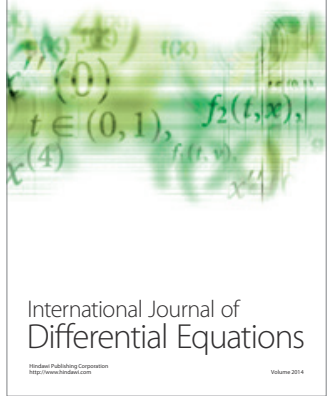
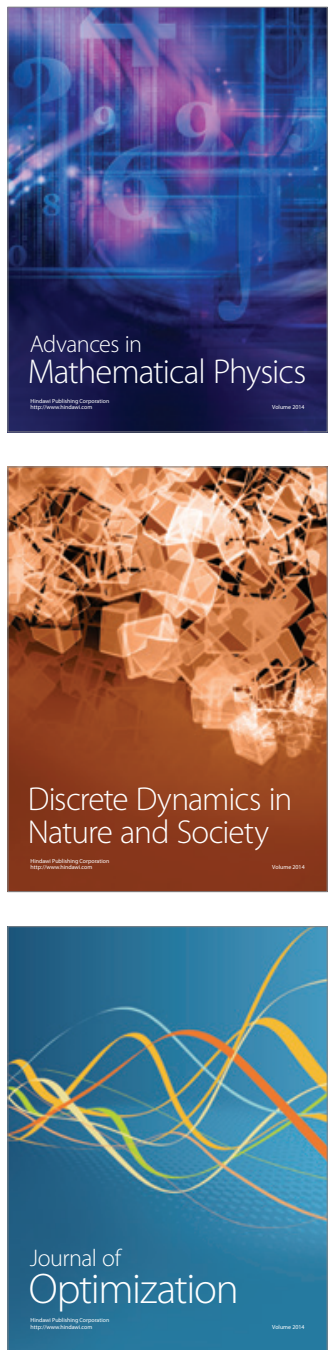